\title{
Traslado de postlarvas de Litopenaeus vannamei (Boone, 1931) a diferentes tiempos, salinidades y densidades y su efecto en la supervivencia y algunos marcadores bioquímicos
}

\author{
Transportation of Litopenaeus vannamei (Boone, 1931) postlarvae at different times, \\ salinities and densities and its effect on their survival and some biochemical markers
Barbarito Jaime-Ceballos ${ }^{1}$, José Galindo-López ${ }^{1}$, Ernesto Laria-Lamela ${ }^{1}$, Fabio Cupul-Magaña ${ }^{2}$ y Fernando Vega-Villasante ${ }^{2}$

\begin{abstract}
${ }^{1}$ Centro de Investigaciones Pesqueras, $5^{\text {ta }}$ Avenida y Calle 246. Barlovento, Santa Fé, Municipio Playa, Ciudad de La Habana, Cuba
${ }^{2}$ Grupo de Investigaciones Costeras. Departamento de Ciencias Biológicas. Centro Universitario de la Costa, Universidad de Guadalajara. Avenida Universidad $N^{\circ}$ 203, Del. Ixtapa, C.P. 48280, Puerto Vallarta, Jalisco, México fvillasante@pv.udg.mx
\end{abstract}

\begin{abstract}
The conditioning of white shrimp Litopenaeus vannamei postlarvae was evaluated during the transfer to tanks at different salinities $(0,5,10$ and $15 \mathrm{psu})$ and two densities (10 and 300 postlarvae $\mathrm{L}^{-1}$ ), during three times (1, 3 and $6 \mathrm{~h}$ ). When the transfer time increased, survival decreased in both densities and mortality rate was higher when a 300 postlarvae $\mathrm{L}^{-1}$ density was used. A significant interrelation was found between both factors, time and salinity. In general, when salinity was lower the survival in transfer times also decreased. Survival increased with a shorter transfer time. Significant differences were found between both transfer densities when salinity
\end{abstract}

increased and greater survival rate was observed at the lowest density (150 postlarvae $\left.\mathrm{L}^{-1}\right)$. Biochemical markers showed significant variations on glucose concentration and phenoloxidase activity, probably due to stress conditions. A pre-acclimatizing time at a salinity level no lower than 5 psu during $48 \mathrm{~h}$ followed by a transfer at the same salinity, with a transfer density lower than 300 postlarvae $\mathrm{L}^{-1}$ and a transfer period no longer than $6 \mathrm{~h}$ is proposed.

Key words: Shrimp culture, salinity, density, glucose, phenoloxidase

\section{Introducción}

El camarón blanco del Pacífico, Litopenaeus vannamei (Boone, 1931), es la especie de camarón más cultivada en el hemisferio occidental (Davis et al. 2004) y, debido a su alta tolerancia a la baja salinidad, ha sido seleccionado como un excelente candidato para ser cultivado en aguas continentales (Bray et al. 1994, Saoud et al. 2003).

El cultivo de camarón marino tierra adentro, fuera de condiciones marinas, es una práctica común en Tailandia (Boyd et al. 2002). Actualmente este tipo de producción ha sido adoptada por varios países americanos como Brasil, Ecuador, EUA, México, Panamá y Venezuela (Jory 2002, Perez \& Garcia 2002, Salame \& Salame 2002, Treece 2002, Balbi et al. 2005). En Cuba se han realizado estudios de cultivo de $L$. vannamei en aguas interiores donde la salinidad puede registrar valores de 0 ups (Jaime \& Galindo 2006).

La disponibilidad regular y estable de postlarvas sanas es uno de los factores de los cuales depende el cultivo comercial de camarón. Sin embargo, con frecuencia, los laboratorios de producción de larvas se encuentran lejos de los estanques de cultivo, por lo cual, los organismos deben ser transportados largas distancias en las mejores condiciones y con el menor estrés posible.

Se debe asegurar la aclimatación de postlarvas en condiciones de alta salinidad a otras de baja salinidad, con el objetivo de alcanzar un cultivo exitoso con bajas tasas de mortalidad (MacGraw et al. 2002, Balbi et al. 2005). Los protocolos de acondicionamiento difieren entre los diferentes semilleros y granjas y están frecuentemente basados en experiencias empíricas (MacGraw et al. 2002).

La concentración de glucosa y la actividad de algunas enzimas como la profenoloxidasa son variables mayormente reconocidas como indicadores asociados al estrés en camarones peneidos (Racotta \& Palacios 1998, Perazzollo et al. 2002).

Hay pocos estudios que aborden el traslado de postlarvas entre los laboratorios de producción y las granjas localizadas tierra adentro. La salinidad, el tiempo de transporte y la densidad larvaria durante el traslado son, entre otros, factores que pueden afectar la supervivencia de las postlarvas. La relación entre estas variables y su efecto sobre la supervivencia de postlarvas de $L$. vannamei durante el traslado es analizada en el presente estudio. 


\section{Material y métodos}

El diseño experimental consistió en 24 tratamientos donde se incluyeron todas las posibles combinaciones de las variables tiempo de traslado (T) $(1,3$ y 6 h), densidad (D) de postlarvas (PL) (150 y $\left.300 \mathrm{PL} \mathrm{L}^{-1}\right)$ y la salinidad del agua $(\mathrm{S})(0,5,10$ y 15 ups). Cada tratamiento fue realizado por triplicado.

Las $\mathrm{PL}_{20}$ de L. vannamei fueron adaptadas (de acuerdo con el protocolo de la compañía cubana GEDECAM) a las diferentes salinidades experimentales, $48 \mathrm{~h}$ antes del traslado. La salinidad del agua fue ajustada (oxímetro multifuncional YSI85 (C) diluyendo agua marina (35 ups) con agua de pozo (0,3 ups). Durante este tiempo los organismos fueron alimentados cada dos horas, de acuerdo con el protocolo de alimentación de la compañía cubana GEDECAM (Tabla 1). La calidad de las PL fue evaluada de acuerdo a la prueba de estrés de Davis et al. (2004).

Al agua de cultivo y de transporte se le adicionó EDTA-Na ${ }_{2}\left(10 \mathrm{mg} \mathrm{L}^{-1}\right)$. La temperatura y el oxígeno disuelto del agua durante el traslado fue de $22,30 \pm 0,05^{\circ} \mathrm{C}$ y $5,32 \pm$ $0,48 \mathrm{mg} \mathrm{L}^{-1}$, respectivamente. El traslado de las PL se realizó en bolsas de plástico con agua saturada de oxígeno. Durante el traslado las larvas fueron alimentadas con hojuelas $\left(0,03 \mathrm{mg} \mathrm{PL}^{-1}\right)$.

Con el objetivo de determinar el efecto en marcadores bioquímicos seleccionados de las diferentes salinidades establecidas antes del traslado, la concentración de proteína, glucosa y calcio fue cuantificada, así como la actividad de fenoloxidasa (PO) y peroxidasa (POD), a través de la siguiente metodología: antes del traslado, un grupo de $0,1 \mathrm{~g}$ de postlarvas aclimatadas durante $48 \mathrm{~h}$ fue seleccionado al azar y almacenado hasta su utilización $\left(-20^{\circ} \mathrm{C}\right)$; las muestras fueron homogenizadas con tampón fosfato salino (PBS), $\mathrm{pH} 7,0$, centrifugadas (10000 g 5 $\min ^{-1}$ ) y el sobrenadante almacenado a $-20^{\circ} \mathrm{C}$ hasta $\mathrm{su}$ uso. La concentración de proteína fue cuantificada de acuerdo con Henry (1964), la concentración de glucosa de acuerdo con el Kit Rapigluco-test de HELFA Diagnostics $®$, la concentración de calcio de acuerdo con

\section{Tabla 1}

Programa de alimentación de las postlarvas de L. vannamei durante las 48 h de aclimatación

Feeding schedule of L. vannamei postlarvae during $48 \mathrm{~h}$ acclimation

\begin{tabular}{lccc}
\hline Tipo de alimento & Origen & $\begin{array}{c}\text { Proteína } \\
(\%)\end{array}$ & $\begin{array}{c}\text { Ración } \\
\left(\mathrm{g} \mathrm{m}^{-3}\right)\end{array}$ \\
\hline Biomasa de Artemia & Ecuador & 36 & 2,7 \\
Migaja $(800-1.500 \mu \mathrm{m})$ & Malta Texo Co. & 35 & 0,22 \\
Hojuela & Salt Creek, INC & 53 & 0,4 \\
ABM 4.000 $(400 \mu \mathrm{m})$ & Bio-marine, INC & 50 & 0,5 \\
\hline
\end{tabular}

la prueba de calcio de Centis Diagnosticus ${ }^{\circledR}$, la actividad de PO de acuerdo con Barraco \& Söderhäll (1996) y la actividad de POD de acuerdo con Welinder (1979).

Una vez concluido cada traslado, el número de postlarvas fue cuantificado para calcular el porcentaje de supervivencia. De igual forma se recolectó $0,1 \mathrm{~g}$ de postlarvas seleccionadas al azar de cada tratamiento que fueron almacenadas hasta su utilización $\left(-20^{\circ} \mathrm{C}\right)$. Posteriormente se determinó el efecto de los tratamientos de traslado sobre determinados marcadores bioquímicos siguiendo la metodología ya mencionada. Los valores de supervivencia fueron transformados a arco seno «p; la normalidad y homocedasticidad de los datos fueron evaluadas utilizando las pruebas de Kolmogorov-Smirnov y Bartlett, respectivamente (Sheskin 2007). Un modelo estadístico trifactorial fue aplicado con las variables $\mathrm{T}, \mathrm{S}$ y D de traslado y tres, cuatro y dos niveles por cada variable respectivamente. Posteriormente se aplicó la prueba de Holm-Sidak (Glantz 2005) para comparación múltiple de medias entre las interacciones y las variables. El manejo estadístico de los datos fue hecho con el programa Sigma Stat $3.1^{1}$.

\section{Resultados y discusión}

El análisis estadístico demostró un efecto de interacción entre T-S, T-D y D-S sobre la supervivencia. No se encontraron diferencias en la concentración de proteína de las postlarvas como resultado del efecto de los diferentes tratamientos.

La Tabla 2 muestra el efecto del tiempo de traslado y la densidad de PL sobre la supervivencia. Con un traslado de una hora, la supervivencia no fue afectada por la densidad. Hubo diferencias significativas en supervivencia entre ambas densidades a las $3 \mathrm{~h}$ de traslado. El traslado de seis horas incrementó la mortalidad en ambas densidades, y la menor supervivencia fue registrada en $300 \mathrm{PL} \mathrm{L}^{-1}$.

La Tabla 3 muestra el efecto del tiempo de traslado y la salinidad del agua. Con una hora de traslado la supervivencia no fue afectada por la salinidad del agua. Un efecto negativo sobre la supervivencia se observó a las tres horas de traslado en agua con 0 ups. La supervivencia de PL en seis horas de traslado disminuyó en agua con 0,5 y 10 ups.

La Tabla 4 muestra la relación entre la densidad de PL y la salinidad de agua durante el traslado sobre la supervivencia de las postlarvas. Salinidades menores disminuyeron significativamente la supervivencia de PL

\footnotetext{
${ }^{1}$ (Systat Software, Inc. Point Richmond, CA 94804-2028, USA).
} 
Tabla 2

Efecto del tiempo y la densidad de traslado en la supervivencia de postlarvas (PL) de $L$. vannamei. Cada valor representa el promedio \pm desviación estándar $(\mathrm{n}=12)$

Transfer time and density effect over L. vannamei postlarval (PL) survival. Each value represents the average \pm standard deviation $(n=12)$

\begin{tabular}{llccc}
\hline & & \multicolumn{3}{c}{ Tiempo (h) } \\
\cline { 3 - 5 } & & 1 & 3 & 6 \\
\hline \multirow{2}{*}{ Densidad $\left(\mathrm{PL} \mathrm{L}^{-1}\right)$} & 150 & $97,93 \pm 2,89^{\mathrm{a}}$ & $99,39 \pm 0,99^{\mathrm{a}}$ & $82,18 \pm 14,68^{\mathrm{c}}$ \\
& 300 & $99,68 \pm 0,45^{\mathrm{a}}$ & $93,68 \pm 8,17^{\mathrm{b}}$ & $66,94 \pm 28,20^{\mathrm{d}}$ \\
\hline
\end{tabular}

Exponentes diferentes representan diferencias significativas $(P<0,05)$

Tabla 3

Efecto del tiempo de traslado y la salinidad del agua sobre la supervivencia de postlarvas de L. vannamei. Cada valor representa el promedio \pm desviación estándar $(n=6)$

Transfer time and water salinity effect over L. vannamei postlarval survival. Each value represents the average \pm standard deviation $(n=6)$

\begin{tabular}{ccccc}
\hline & & \multicolumn{3}{c}{ Tiempo $(\mathrm{h})$} \\
\cline { 3 - 5 } & & 1 & 3 & 6 \\
\hline & 0 & $99,28 \pm 0,72^{\mathrm{ab}}$ & $90,52 \pm 11,01^{\mathrm{c}}$ & $49,32 \pm 17,35^{\mathrm{f}}$ \\
Salinidad & 5 & $98,88 \pm 2,74^{\mathrm{ab}}$ & $98,43 \pm 1,96^{\mathrm{b}}$ & $58,73 \pm 13,83^{\mathrm{e}}$ \\
(ups) & 10 & $97,17 \pm 3,08^{\mathrm{b}}$ & $98,38 \pm 1,89^{\mathrm{b}}$ & $92,4 \pm 6,00^{\mathrm{d}}$ \\
& 15 & $99,88 \pm 0,29^{\mathrm{a}}$ & $98,82 \pm 1,12^{\text {ab }}$ & $97,8 \pm 1,82^{\mathrm{b}}$ \\
\hline
\end{tabular}

Exponentes diferentes representan diferencias significativas $(P<0,05)$

\section{Tabla 4}

Efecto de la densidad y la salinidad del agua durante el traslado sobre la supervivencia de postlarvas (PL) de

L. vannamei. Cada valor representa el promedio \pm desviación estándar $(n=9)$

Postlarval (PL) density and water salinity effect over

L. vannanei survival. Each value represents the average \pm standard deviation $(n=9)$

\begin{tabular}{cccc}
\hline & \multicolumn{2}{c}{ Densidad $\left(\mathrm{PL} \mathrm{L}^{-1}\right)$} \\
\cline { 2 - 4 } & & 150 & 300 \\
\hline \multirow{3}{*}{ Salinidad } & 0 & $87,11 \pm 17,63^{\mathrm{c}}$ & $72,3 \pm 29,96^{\mathrm{e}}$ \\
(ups) & 10 & $89,56^{\mathrm{b}} \pm 14,41^{\mathrm{c}}$ & $73,53 \pm 26,00^{\mathrm{d}}$ \\
& 15 & $96,3 \pm 5,14^{\mathrm{b}}$ & $95,6 \pm 4,35^{\mathrm{b}}$ \\
& $99,63 \pm 0,58^{\mathrm{a}}$ & $98,02 \pm 1,66^{\mathrm{b}}$ \\
\hline
\end{tabular}

Exponentes diferentes representan diferencias significativas $(P<0,05)$ a ambas densidades. Altas densidades afectaron la supervivencia cuando la salinidad fue inferior a 10 ups.

La Fig. 1 muestra el efecto de 48 h de aclimatación de PL a diferentes salinidades sobre la actividad de POD y PO. No se encontraron diferencias significativas en la actividad de POD con las salinidades probadas. La actividad de PO disminuyó significativamente en organismos expuestos a agua con salinidades de 0 y 5 ups, en comparación con los expuestos a mayores salinidades.

La Fig. 2 muestra el efecto de 48 h de aclimatación de PL a diferentes salinidades sobre la concentración de glucosa y calcio. No se encontraron diferencias significativas en la concentración de calcio a las salinidades probadas. La concentración de glucosa se incrementó en los organismos expuestos a agua con salinidades de 0 y 5 ups. 


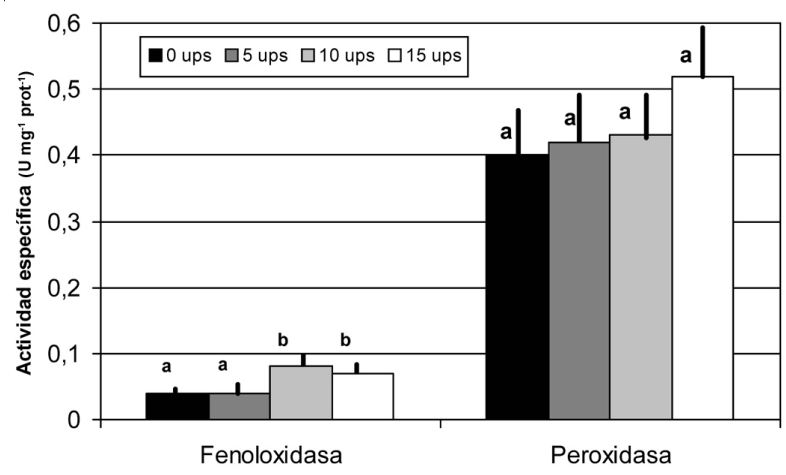

Figura 1

Efecto de la aclimatación de postlarvas de $L$. vannamei durante $48 \mathrm{~h}$ a diferentes salinidades sobre la actividad de PO y POD. Letras diferentes muestran diferencias estadísticas $(P<0,05)$. Diferentes letras muestran diferencias estadísticas $(P<0,05)$. Cada valor representa el promedio \pm desviación estándar $(\mathbf{n}=10)$

Effect of 48-h acclimation at different salinities of $L$. vannamei postlarvae over PO and POD activities. Different letters show statistical differences $(P<0.05)$. Each value represents the average \pm standard deviation $(\mathrm{n}=10)$

Durante el traslado la actividad de PO fue afectada por los factores individuales T, D y S y los factores combinados $\mathrm{T}$ y D. La concentración de glucosa se incrementó de acuerdo con la densidad de traslado (datos no mostrados).

El cultivo de camarón en granjas tierra adentro requiere un proceso de aclimatación de PL. Las PL son usualmente obtenidas de semilleros y transferidas en agua con salinidades de 28 a 35 ups (salinidad de agua oceánica). Las PL deben ser aclimatadas a las salinidades ambientales (y perfiles iónicos) de los estanques de crecimiento receptores, antes de ser transferidas a los mismos (McGraw et al. 2002). Dos estrategias de aclimatación son comúnmente usadas por los productores de camarón. Una de ellas es una estancia temporal seguida de una aclimatación de corta duración (menor a $8 \mathrm{~h}$ ), la segunda involucra una estancia más larga seguida por una aclimatación lenta (Davis et al. 2004).

La etapa de mayor tolerancia a grandes fluctuaciones de salinidad para la mayoría de los peneidos es entre PL10 y PL40 (Tsuzuki et al. 2000), tal hecho limita el periodo en que un productor puede mantener a los camarones antes de llevar a cabo su aclimatación a aguas con menores niveles de salinidad.

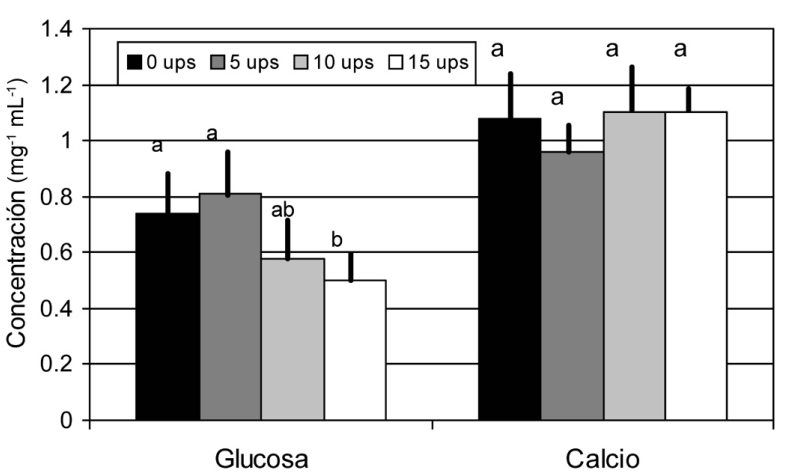

Figura 2

Efecto de la aclimatación de postlarvas de $L$. vannamei durante $48 \mathrm{~h}$ a diferentes salinidades, sobre la concentración de glucosa y calcio. Distintas letras muestran diferencias estadísticas $(P<0,05)$.

Cada valor representa el promedio \pm desviación estándar $(\mathrm{n}=10)$

Effect of 48-h acclimation at different salinities of L. vannamei postlarvae over glucose and calcium concentration. Different letters show statistical differences $(P<0.05)$. Each value represents the average \pm standard deviation $(n=10)$

Tanto en el proceso de aclimatación como en el crecimiento uno de los mayores problemas del cultivo es la composición iónica del agua y no sólo su salinidad (Balbi et al. 2005). Los camarones requieren agua con concentraciones específicas de los aniones más importantes: bicarbonatos, sulfatos y cloruros; y de los cationes más importantes: calcio, magnesio, potasio y sodio (Boyd et al. 2002).

En nuestros resultados, bajo el protocolo experimental establecido, el tiempo de traslado afectó la supervivencia a las dos densidades evaluadas, con mayores mortalidades con $300 \mathrm{PL} \mathrm{L}^{-1}$. De manera general, la supervivencia disminuyó a las más bajas salinidades durante el traslado, mejores supervivencias fueron obtenidas con tiempos cortos de traslado.

Durante la aclimatación de 48 h y durante el traslado de las postlarvas, algunos marcadores bioquímicos (glucosa y PO) fueron afectados. El efecto individual de los factores tiempo, densidad y salinidad y los combinados tiempo y densidad, están relacionados con la concentración de la $\mathrm{PO}$ en las postlarvas después del traslado. Se registró el incremento de la concentración de glucosa cuando la densidad de postlarvas se incrementó. 
Perazzolo et al. (2002) reportaron que la actividad específica de PO, después de un tratamiento con tripsina, se incrementó significativamente en el suero de machos a los que se les extirpó el espermatóforo, sin embargo, no se encontró una correlación clara entre la actividad de PO y la salinidad del agua. La actividad de PO (total y específica) disminuyó de manera importante en todas las salinidades probadas sugiriendo una supresión parcial del sistema profenoloxidasa posiblemente debida al estrés causado por el cautiverio.

Chanratchakool et al. (1998) $)^{2}$ estudiaron el efecto de cambios rápidos de la temperatura del agua y el $\mathrm{pH}$ en la respuesta inmune del camarón y encontraron que el incremento de la temperatura ambiental resulta en una reducción significativa de la actividad bactericida y de PO. Además, al reducir el pH del agua de 7,8 a 6,0 durante tres horas, se observó una reducción en la actividad de PO, fagocitosis y actividad bactericida. De acuerdo con Pascual et al. (2003), el estrés reduce la capacidad inmunológica a través de la reducción del conteo de hemocitos y alterando los mecanismos reguladores de profenoloxidasa.

Re et al. (2004) mencionan que juveniles de Litopenaeus stylirostris acondicionados a bajas salinidades (10 ups) incrementaron su tasa de excreción de amonio y relacionan esto con condiciones de estrés que conducen a un catabolismo de aminoácidos para la producción de energía necesaria para enfrentar el choque hipo-osmótico. En esta especie, ellos recomiendan desarrollar los cultivos a salinidades no menores de 25 ups.

En nuestros resultados observamos una disminución de la actividad de PO en las postlarvas, relacionada con las diferentes concentraciones salinas del agua. Bajas salinidades resultaron en una menor actividad enzimática probablemente debido, tal y como mencionan los resultados antes mencionados por otros autores, al estrés causado por un ambiente hipo-osmótico.

Por otro lado, los niveles de glucosa de las postlarvas se incrementaron después de $48 \mathrm{~h}$ de aclimatación, sugiriendo un comportamiento metabólico relacionado con el estrés. De acuerdo con Claybrook (1983) y Racotta \& Palacios (1998) cuando los camarones están estresados, la glucosa de la sangre se incrementa para ser usada como una fuente rápida de energía. Bajo tales circunstancias

${ }^{2}$ Chanratchakool P, N Sukrakanchana \& S Pancharatana. 1998. The effects of environmental change on the immune response of the shrimp (Penaeus monodon Fabricius). $6^{\text {th }}$ Kasetsart University Annual Conference, 3-5 February 1998, Tailandia. (metabolismo de huída), los niveles de glucosa sanguínea se pueden elevar sobre sus niveles basales.

De acuerdo con nuestros resultados y otros mencionados, concluimos que la aclimatación y traslado a bajas salinidades produce un serio estrés en las postlarvas que de hecho puede afectar la supervivencia y la salud. Cuando la aclimatación es seguida de traslado, varios factores deben ser tomados en cuenta: tiempo de traslado, densidad de postlarvas durante el traslado y la salinidad de agua de traslado. Davis et al. (2004) sugieren una preaclimatación en los semilleros seguida de una aclimatación final en las instalaciones de engorde, mencionan de igual forma que el traslado de postlarvas puede ser un factor de estrés que a su vez puede tener efectos negativos en la viabilidad y supervivencia de los organismos, sin embargo, no sugieren condiciones de traslado.

Aún y cuando no se realizaron otros bioensayos de preaclimatación previa al traslado, basados en los resultados expuestos en este trabajo se propone un proceso de preaclimatación con disminución de la salinidad a no menos de 5 ups durante $48 \mathrm{~h}$, seguido de un traslado a esta misma salinidad, con densidades de postlarvas menores a $300 \mathrm{PL} \mathrm{L}^{-1}$, y un tiempo de traslado no mayor de $6 \mathrm{~h}$. En las instalaciones de crecimiento, la salinidad puede ser ajustada a niveles menores utilizando la misma agua que será utilizada en los estanques de engorda. Una lenta aclimatación usando la misma agua de los estanques de engorda puede ser benéfica para la viabilidad de las postlarvas, considerando que el perfil iónico (y no sólo la salinidad) del agua de traslado puede ser diferente a la del agua de los estanques de crecimiento.

\section{Agradecimientos}

Los autores desean expresar su agradecimiento a los evaluadores de nuestro manuscrito; sin los atinados comentarios que nos hicieron llegar no hubiera sido posible su publicación. Se agradece también a la M.C. Olimpia Chong, por colaborar en la corrección de las primeras versiones del documento.

\section{Literatura citada}

Balbi F, J Rosas, A Velásquez, T Cabrera \& C Maneiro. 2005. Aclimatación a baja salinidad de postlarvas del camarón marino Litopenaeus vannamei (Boone, 1931) provenientes de dos criaderos comerciales. Revista de Biología Marina y Oceanografía 40: 109-115.

Barraco M \& K Söderhäll. 1996. Effect of fixed epimastigote forms of Trypanosoma cruzi on the hemocyte and the prophenoloxidase activating system of the crayfish Pacifastacus leniusculus. Brazilian Journal of Medical and 
Biological Research 29: 1321-1327.

Boyd C, T Thunjai \& M Boonyaratpalin. 2002. Dissolved salts in water for inland low-salinity shrimp culture. Global Aquaculture Advocate 5: 40-45.

Bray WA, AL Lawrence \& JR Leung-Trujillo. 1994. The effect of salinity on growth and survival of Penaeus vannamei, with observations on the interaction of IHHN virus and salinity. Aquaculture 122: 133-146

Claybrook DL. 1983. Nitrogen metabolism. En: Mantel LH (ed), The biology of Crustacea, internal anatomy and physiological regulation. Vol. 5: 163-213. Academic Press, Nueva York.

Davis DA, TM Samocha \& CE Boyd. 2004. Acclimating Pacific white shrimp Litopenaeus vannamei to inland lowsalinity waters. Southern Regional Aquaculture Center. SRAC Publication No 2601 [on-line] < http:// www.ca.uky.edu/wkrec/2601fs.PDF>

Glantz SA. 2005. Primer of Biostatistics. 203 pp. McGrawHill, Nueva York.

Henry RJ. 1964. Colorimetric determination of the total protein. En: Henry RJ (ed). Clinical Chemistry, Principles and Technics, p.181. Harper \& Row, Nueva York.

Jaime B \& J Galindo. 2006. Cultivo del camarón blanco Litopenaeus vannamei a baja salinidad: traslado de postlarvas. CD de Memorias del XVI Forum de Ciencia y Técnica. Ministerio de la Industria Pesquera, Cuba.

Jory D. 2002. Inland shrimp culture with zero water exchange ponds. Aquaculture Magazine 28(5): 74-77.

McGraw W, D Davis, D Teichert-Coddington \& D Rouse. 2002. Acclimation of Litopenaeus vannamei postlarvae to low salinity: Influence of age, salinity endpoint, and rate of salinity reduction. Journal of the World Aquaculture Society 33: 78-84.

Pascual C, A Sánchez, A Sánchez, F Vargas-Albores, G LeMoullac \& C Rosas. 2003. Haemolymph metabolic variables and immune response in Litopenaeus setiferus adult males: the effect of an extreme temperature. Aquaculture 218(1-4): 637-650.
Perazzolo L, R Gargioni, P Ogliari \& MAA Barraco. 2002. Evaluation of some hemato-immunological parameters in the shrimp Farfantepenaeus paulensis submitted to environmental and physiological stress. Aquaculture 214(14): 19-33.

Pérez H \& C Garcia. 2002. Freshwater trial white Litopenaeus vannamei leads to further stocking in Panama. Global Aquaculture Advocate 5: 39.

Racotta IS \& E Palacios. 1998. Hemolymph metabolic variables in response to experimental manipulation stress and serotonin injection in Penaeus vannamei. Journal of the World Aquaculture Society 29: 351-356.

Re AD, F Díaz, E Sierra \& S Gómez-Jiménez. 2004. Consumo de oxígeno, excreción de amonio y capacidad osmorreguladora de Litopenaeus stylirostris (Stimpson) expuesto a diferentes combinaciones de temperatura y salinidad. Ciencias Marinas 30: 443-453.

Salame M \& A Salame. 2002. Inland shrimp farming in Ecuador - The Inacua Experience. Global Aquaculture Advocate 5: 48-49.

Saoud IP, DA Davis \& DB Rouse. 2003. Suitability studies of inland well waters for Litopenaeus vannamei culture. Aquaculture 217: 373-383.

Sheskin D. 2007. Handbook of parametric and nonparametric statistical procedures, pp. 241-255. Chapman \& Hall, CRC, Boca Raton.

Treece G. 2002. Inland shrimp farming in west Texas, U.S.A. Global Aquaculture Advocate 5: 46-47.

Tsuzuki MY, RO Cavalli \& A Bianchini. 2000. The effects of temperature, age, and acclimation to salinity on the survival of Farfantepenaeus paulensis postlarvae. Journal of the World Aquaculture Society 31(3): 459-468.

Welinder KG. 1979. Amino acid sequence studies of horseradish peroxidase: amino and carboxyl termini, cyanogen bromide and tryptic fragments, the complete sequence, and some structural characteristics of horseradish peroxidase C. European Journal of Biochemistry 96: 483502. 